\title{
Improving the Accuracy of Reliability Models for Direct Interconnection Networks:
}

\author{
Rosa Alcover ${ }^{1}$, Vicente Chirivella ${ }^{1}$, and José Duato ${ }^{2}$ \\ ${ }^{1}$ Department of Statistics and Operation Research, \\ \{ralcover, vchirive\}@eio.upv.es \\ ${ }^{2}$ Department of Information Systems and Computer Architecture \\ Polytechnic University of Valencia, \\ Camino de Vera s/n, 46020 Valencia, Spain \\ jduato@gap.upv.es
}

\begin{abstract}
Fault-tolerance in multicomputer interconnection networks has been traditionally studied by determining the worst possible combination of faulty components that causes a network failure and then assuming that this will occur. But, the worst possible combination may occur with low probability and the routing algorithm may allow the network to work, even when there is a large number of faults. Thus, the network dependability parameters computed according to this approach will be underestimated. In a previous paper [3], we have proposed a new methodology, based on Markov chains, for evaluating interconnection network dependability. Using this methodology, we can accurately compute the network reliability behavior. In this paper we apply it to evaluate dependability parameters in a 2-D mesh, taking into account network size, routing algorithm, failure and repair rates of nodes and coverage. Finally, we compare the computed results under traditional and our approach.
\end{abstract}

\section{Introduction}

Day by day, many scientific disciplines demand more computing power. These demands should be satisfied by new generations of multicomputers constructed with larger number of processors. As the number of elements in the interconnection networks increases with the number of processing units, the probability of component failures increases too. Therefore, the network fault-tolerance properties are a key issue in determining the overall performance of these machines. Dependability [2] analysis of multicomputers is essential to evaluate their effectiveness for critical and real time applications.

The objective is to design an interconnection network that works in the presence of faulty components. Fault-tolerant routing algorithms allow to achieve this objective [5], [7] because they bypass faulty components, taking advantage of the alternative

\footnotetext{
* This work was supported by the Polytechnic University of Valencia under Grant 6645.
} 
paths existing in the network. However, the maximum number of faults supported by these algorithms is bounded [5].

On the other hand, although many fault-tolerant routing algorithms have been proposed, hardly any one has been implemented in commercial machines. Nevertheless, some commercial machines implement simple fault-tolerant routing algorithms, for instance, the Cray T3E [8]. When fault-tolerant routing algorithms are defined, the authors determine the maximum number of failures that the algorithms can support. This number corresponds to the worst possible combination of failure positions that leads to the network failure [7]. Of course, this number is bounded by the interconnection network topology. For these cases, very few papers analyze the behavior of the network when the number of faults goes beyond the limits imposed by topology. The only studies we know about this have been performed from an experimental point of view, by randomly generating sets of faulty components [4], [7], [9]. As a consequence, there is not a clear idea about the system reliability based on those routing algorithms. This is the traditional approach. However, the worst possible combination does not always occur, and the routing algorithm is usually able to route in the presence of a larger number of failures. This fact has not been considered in previous papers and is the focus of our research.

In a previous paper [3] we proposed an analytical methodology for reliability and availability prediction of interconnection networks based on continuous-parameter Markov chains [2]. This methodology takes into account the topology, network size and the routing algorithm used, and allow us to measure the effect of the routing algorithm in the interconnection network reliability.

In this paper, we apply this methodology to evaluate dependability parameters in a mesh. Also, we measure the differences between the traditional approach and a more accurate one, in connection with these parameters. We summarize this methodology in section 2 . Then, in section 3 we apply it to compute the reliability function and the mean time to failure for an interconnection network with mesh topology, wormhole switching and Duato's Double East Last West Last (DELWL) fault tolerant routing algorithm [5].Also, we propose a model to compute the reliability function and the mean time to failure for an interconnection network, and we compare the obtained results with both approaches. We show that network reliability parameters obtained with the traditional approach are always underestimated. Finally, in section 4 some conclusions are drawn in connection with our previous paper.

\section{A Basic Dependability Methodology Based on Markov Chains}

In our previous paper [3] we proposed the basic dependability methodology. It can be briefly summarized in the following steps:

1- Define the Interconnection Network Fault Model.

2 - Select the network dependability parameters.

3 - Define the network states and the transition rates.

4 - Compute the values of the network reliability parameters.

5 - Analyze the results. 


\section{Applying the Dependability Methodology}

In this section we apply the analytical methodology summarized in section 2 . We also propose two models to compute the reliability function $(R(t))$ and the mean time to failure $(M T T F)$ for a network with mesh topology. One model is proposed from the traditional point of view and the other under our approach.

Step 1. The interconnection network fault model includes the network selection and the establishment of the assumed hypothesis about its operation.

As in our previous paper, the interconnection network selected is a mesh, with wormhole switching as flow control mechanism, and the DELWL fault-tolerant routing algorithm. Unlike our previous paper, channels are reliable and only the node failures are studied in this paper.

Concerning to the hypothesis assumed in the network fault model, we suppose that a network fails when a node cannot communicate with another one, either because there are no physical links between them, or because the routing algorithm cannot select a route to reach the destination node. We also assume that if there is a selectable path between two nodes, the path will be selected. Some faulty node positions combination may disconnect the network. If the network is disconnected, the network fails. A failed node simply ceases to work, and the channels connected to a malfunctioning node are not selected by the routing algorithm.

Fault-tolerant routing algorithms automatically recover the system from the occurrence of some node failures during normal operation. The recovery consists on the detection of the fault, the identification of the faulty node, the correction of the errors induced by the fault, and notification to the neighboring nodes that there is a faulty node in the network. However, fault detection mechanisms can fail with a certain probability. The probability of system recovery when a fault occurs is called coverage, $C$ [6]. This probability is assumed to be constant. Finally, the network is repairable, either after a complete failure, or after the partial failure of some nodes.

The modeling of network operation has been based on continuous-parameter Markov chains. We have considered exponential distribution with $\lambda$ parameter for the nodes failure times, with $\mu$ parameter for the nodes reparation times, and a uniform distribution of message destinations. The mean time to node failure is measured in months, its typical value being between 1 and 6 months. The lowest value corresponds to machines mounted in rooms and wired externally, while the highest one corresponds to multicomputers assembled in cabinets. The mean time to node repair is measured in hours. We have considered 3, 6, 12, 24, 48 and 72 hours. The lowest value corresponds to military applications, while the highest values correspond to non-critical applications. Finally, the chosen values for $C$ are 0'95, 0'99 and 0'999.

Step 2. In this step, we must select the parameters that will quantify the dependability characteristics that we want to study. For the sake of clarity, in this paper we have selected the reliability function $R(t)$, and the mean time to failure MTTF [1].

Step 3. Now, we define the Markov chains used for modeling the two approaches proposed in this paper. For this, we must specify the states that represent the network operation and establish the transitions between them. First, we define the model from the traditional approach. Then, we will do the same with our approach, and will pay special attention to the expressions of the transition rates between states. 
A Markov chain consists of a set of states and a set of labeled transitions between the states. In our paper, we define the states taking into account the MTTF and according to the number of faulty nodes in the network. Thus, we have considered the following states in the models: Correct State, when there is no faulty node; Degraded State, when there is a faulty node, but the fault detection mechanisms have detected the fault and the routing algorithm can transmit messages between any pair of nodes; and Failed State, when the failure of the network occurs, whatever its cause is.

After a sojourn in a state, the network state will make a transition to another state, with a certain probability, according to its functioning. Transition rates are the change with time of the probability of transit from one state to another state. With the previous states, we propose two models (shown in Figure 1) to compute the network reliability function. The model on the left corresponds to the traditional approach, while the other one fits to our approach. The traditional approach to fault-tolerant routing on a 2-D mesh assumes that the routing algorithm can support a single failure; that is, the network fails with the failure of the second node (worst combination). Thus, the transition rates among states depend on the size of the mesh, but not on the algorithm used for routing. As it is considered that the algorithm cannot route after the second failure, there are no differences among fault-tolerant routing algorithms proposed by different authors.

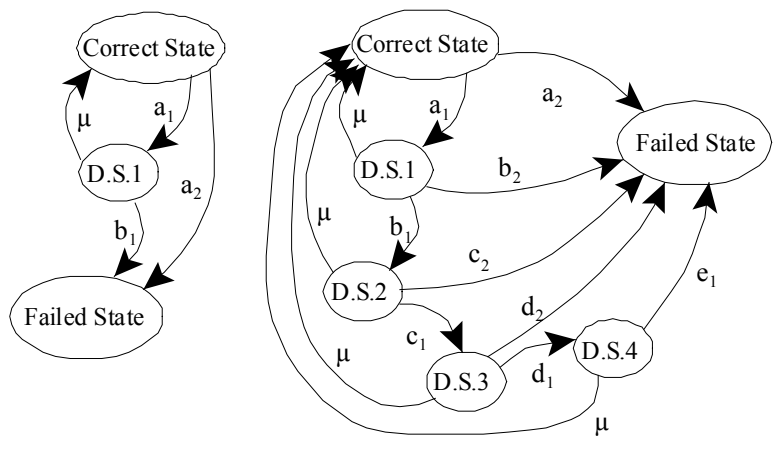

Fig. 1. Reliability models. Traditional (left) and new approach (right)

The model on the left in Figure 1 allows us to obtain $R(t)$. From the Correct State, the network changes to the Degraded State, D.S.1 (transition rate al) if a failure occurs and the fault is covered, or to the Failed State (a2) if the fault is uncovered. In the D.S.1, the network changes to the Correct State if repaired $(\mu)$ or to the Failed State when the next failure occurs (bl). For example, in a 16x16 mesh, the transition rate $a l$ is the product of the number of nodes (256), the coverage and the node failure rate $(a 1=256 C \lambda) ; a 2$ is the product of the number of nodes, the failure probability in the fault detection mechanism and the node failure rate $(a 2=256(1-C) \lambda) ; b 1$ is the product of the number of remaining non-faulty nodes and the node failure rate $(b l=255 \lambda)$.

As the algorithm can route messages if certain combinations of failure locations occur, the transition probabilities will depend on the fault-tolerant routing algorithm chosen and on the mesh size. The state diagram on the right in Figure1, shows the model when our approach is used. The network starts in the Correct State and evolves to the Degraded State when a fault occurs if the failure is covered and, the routing 
algorithm can still establish communication between any pair of nodes. The network changes to the Failed State if at least one of the two conditions fails. The difference with the previous model is that from a Degraded State, the network can reach another Degraded State. The transition rate between the two states depends on the ability of the routing algorithm to maintain communication after a new failure. We consider that after the fifth faulty node, the network performance are too low. Thus, after the D.S.4 the network evolves to the Failed State.

The evolution of the network state could be explained as follows. The network initial state is the Correct State. As failures occur, the network state evolves into the final Degraded States, if the faults are covered, or to the Failed State if the fault is not covered. When $\lambda$ is small, the network spends most of the time in the Correct State and the initial Degraded States, specially when $C$ is high. This is due to the long time to network failure and the high repair rate. If the network is repaired quickly, the network will usually be in the Correct State, and it will move away from this state as time to repair increases. If $C$ is high, the network will tend to move to the degraded states and there will be more opportunities to repair the network. The failure of the network usually occurs when a failure is not covered rather than when many connections have failed. As the node failure rate increases, the network state evolves to intermediate degraded states. The network can be repaired and its more frequent states are the Correct State and the intermediate Degraded States, although now the Failed State is reached more frequently. As $C$ increases, the network tends to continue working because it has more opportunities to be repaired. The failure of the network occurs more frequently when the number of node failures reaches the maximum number of failed nodes allowed. When the node failure rate is high, the network quickly evolves to the Failed State. A high value of $\mu$ allows the network to work. Otherwise the network will be repaired only in a very few occasions.

Given a topology, a routing algorithm and a number of faulty nodes, it is necessary to study the combinations of faulty locations that cause the network to fail. Then, the transition rates can be computed. In our model, the expression of the transition rate $q_{i, i+1}$ from the Degraded State $i(i \geq 0)$ to the next Degraded State $i+1$ is given by

$$
q_{i, i+1}=(N-i) r p_{i+1} C \lambda,
$$

and the transition rate from the Degraded State $i$ to the Failed State is given by

$$
q_{i, F . S .}=(N-i)\left(1-r p_{i+1} C\right) \lambda,
$$

with

$$
r p_{i+1}=n f c_{i+1} /\left(n c f_{i}(N-i)\right)
$$

where $N$ is the number of nodes; $r p_{i+1}$ is the probability of network working when a new failure takes place, knowing that $i$ failures have already taken place in an operational network; and $n f c_{i+1}$ is the number of combinations of $i+l$ faulty nodes that do not cause the network failure, knowing that such combinations are obtained from the combinations of $i$ faulty nodes that did not cause the network failure, with the positions of a new faulty node. For example, taking into account the network size (16x16) and the routing algorithm, the transition rate b1 from D.S.1 to D.S.2, computed with equation (1), is now the product of the number of remaining non faulty nodes, the probability of routing after the occurrence of two given failures, the fault coverage and the failure rate of a node. So, the expressions of $b 1$ and $b 2$ are $b 1=$ 
$253^{\prime} 9 C 8$, and from equation $(2), b 2=\left(255-253^{\prime} 9 C\right) 8$. Table 1 shows the transition rates computed according both approaches and the routing algorithm DELWL.

Step 4. Now, the values of the selected dependability parameters must be obtained. So, we must solve the system of differential equations that govern the state probabilities [2]. The expressions of this parameters can be easily obtained. Thus, $R(t)$ for the traditional approach is the sum of the probabilities of being in the Correct State, or in the Degraded State, while in our approach, it is the sum of the probabilities of being in the Degraded States, numbered from 1 to 4, or in the Correct State. With the values of $\lambda, \mu$, and $C$, the MTTF has been computed and the results have allowed us to obtain the plots shown in the next section.

Table 1. Transition rates for both approaches in $5 \times 5$ and $16 \times 16$ meshes

\begin{tabular}{|l|l|l|l|}
\hline \multicolumn{4}{|c|}{ Traditional approach } \\
\hline $\mathbf{5 x 5}$ Mesh & $a 1=258 C$ & $a 2=258(1-C)$ & $b 1=248$ \\
\hline $\mathbf{1 6 x 1 6}$ Mesh & $a 1=256 C 8$ & $a 2=256(1-C) 8$ & $b 1=2558$ \\
\hline \multirow{4}{|c|}{ New approach } \\
\hline \multirow{5}{*}{ Mesh } & $a 1=258 C$ & $b 2=\left(24-21^{\prime} 6 C\right) 8$ & $d 1=11^{\prime} 848 C$ \\
\cline { 2 - 4 } & $a 2=258(1-C)$ & $c 1=16^{\prime} 188 C$ & $d 2=\left(22-11^{\prime} 84 C\right) 8$ \\
\cline { 2 - 4 } & $b 1=21^{\prime} 6 C 8$ & $c 2=\left(23-16^{\prime} 18 C\right) 8$ & $e 1=218$ \\
\hline \multirow{3}{*}{$\mathbf{1 6 x 1 6}$ Mesh } & $a 1=256 C 8$ & $b 2=\left(255-253^{\prime} 9 C\right) 8$ & $d 1=249^{\prime} 5 C 8$ \\
\cline { 2 - 5 } & $a 2=256(1-C) 8$ & $c 1=251^{\prime} 19 C 8$ & $d 2=\left(253-249^{\prime} 5 C\right) 8$ \\
\cline { 2 - 4 } & $b 1=253^{\prime} 9 C 8$ & $c 2=\left(254-251^{\prime} 19 C\right) 8$ & $e 1=2528$ \\
\hline
\end{tabular}

\section{Results}

Step 5. To compare the MTTF between both approaches, we have used the ratio of the $M T T F$ obtained with our approach and the MTTF obtained with the traditional one. The results appear in Figure 2, represented as a function of $\lambda, \mu$, and $C$.

As shown in the plots, the MTTF ratio is always larger than one. The ratio is in the range 1'2 to 60 , that is, the network MTTF obtained with our approach can be up to 60 times larger than the value obtained with the traditional approach. As our model is much more accurate than the traditional one to evaluate dependability parameters, the MTTF obtained with the traditional approach is always largely underestimated.

The differences between both approaches increase with failure coverage. This can be observed in the sequence formed by Figures $2 \mathrm{a}, 2 \mathrm{~b}$, and $2 \mathrm{c}$, and by Figures $2 \mathrm{~d}, 2 \mathrm{e}$, and $2 \mathrm{f}$, which are ordered according to growing $C$ values. The previous fact occurs because the network tends to transit to a Degraded State instead of to the Failed State, and then there are more opportunities to network repair. The difference between both approaches also increases when 8 diminishes and $\mu, C$ increase. The effects of $C$ and network repair diminish as 8 increases. This occurs because the network state evolves quickly to the Failed State, and the importance of the possibility of network repair diminishes. Only when $C$ is high and the repair time is low, the Degraded States are important and they mark the difference between both approaches.

Therefore, the differences between both approaches grow as the size of the network increases. We can see this fact by comparing Figures $2 a$ and $2 d, 2 b$ and $2 e$, and $2 \mathrm{c}$ and $2 \mathrm{f}$. As the size of the mesh increases, it also increases the number of 
network nodes that could fail. Since the network state evolves quickly to the Failed State, the importance of the possibility of network repair diminishes. The difference between both approaches increases when 8 diminishes and $\mu, C$ increase. Once more, only when $C$ is high and the repair time is low, the Degraded States mark the difference between both approaches.

\section{MTTF ratio}

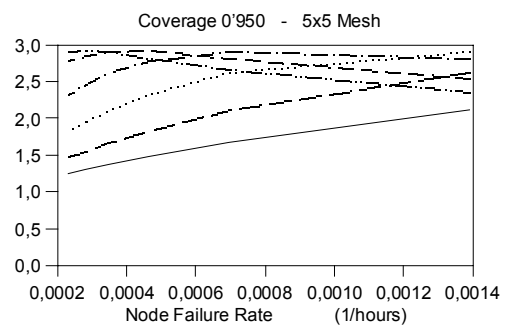

( a )

\section{MTTF ratio}

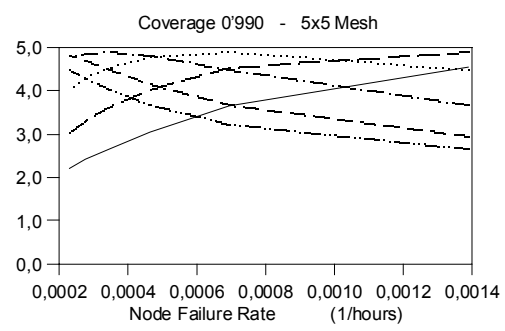

( b )

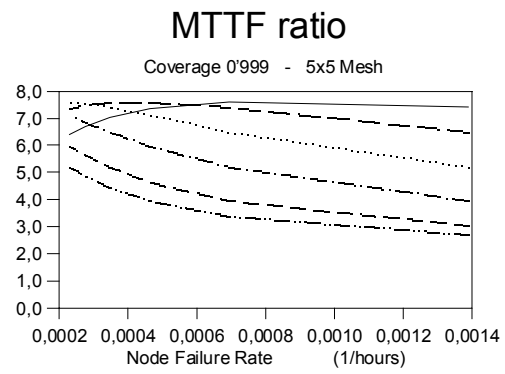

( c )

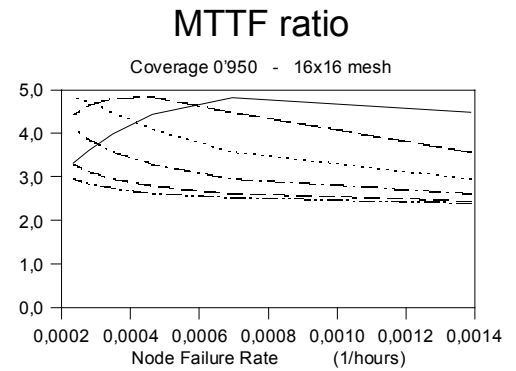

( d )

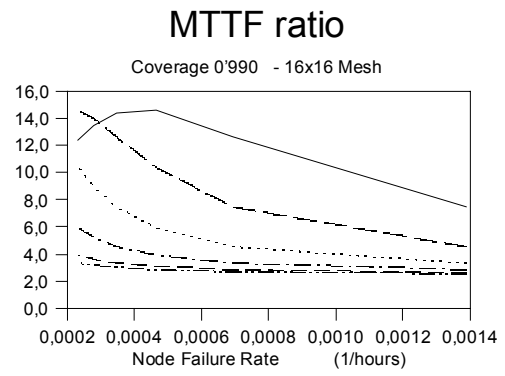

( e )

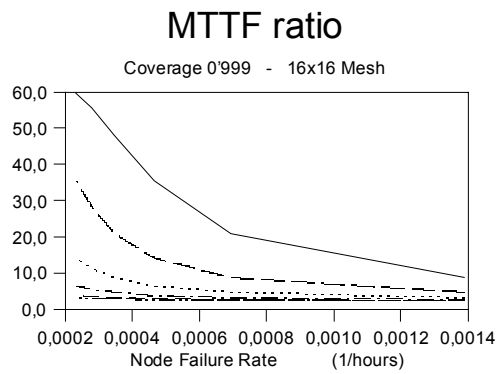

( f )

Mean Time to Repair (hours)

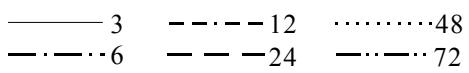

Fig. 2. MTTF ratio as a function of mesh size, coverage and node failure and repair rate

Finally, we can see in the Figure 2 that sometimes the MTTF ratio presents a maximum. This is due to the combination of the effects of the new operational states, Degraded States, and the values of $\delta$ and $\mu$. The importance of these effects can be incremented or diminished by particular values and combination of values of 8 and $\mu$. 


\section{Conclusions}

In this paper we have used a new methodology for computing dependability in interconnection networks based on Markov chains. The Markov chains have allowed us to model the effects of the routing algorithm and the node failure locations in the network. State transitions in Markov chain have been determined by considering the topology (5x5 and 16x16 meshes), the routing algorithm (DELWL) and the number and locations of faulty nodes. This study has taken into account that networks may work even when the number of faulty nodes is larger than the number of faulty nodes supported by the routing algorithm in the worst case. Indeed the main contribution of the proposed model is that they consider all the possible combinations of faults, analyzing their probability and their effects.

We have determined that network reliability parameters computed using the traditional method are too conservative. It must be emphasized that the MTTF is always larger if it is computed according to our approach instead of the traditional approach. The differences are up to 60 times larger, according to the size of the mesh. The differences grow as $C$ increases, $1 / \mu$ is short and $1 / 8$ is long. We have shown that $C$ is the most important parameter on determining the network MTTF.

In a previous paper [3] we studied the effect of channel failure on the MTTF, considering the same interconnection network topology and routing algorithm. Taking into account both papers, we conclude that the differences between both approaches are larger for faulty channels than for faulty nodes. When the mesh size grows, this relationship becomes the opposite.

Finally, our approach is closer to reality than previous approaches because it fits better the network behavior, and consequently provides more accurate values of network dependability parameters.

\section{References}

1. Beaudry M. D.: Performance-Related Reliability Measures for Computing Systems, IEEE Transactions on Computers (1978), vol.27, no.6, 540-547.

2. Bolch, G., Greiner, S., de Meer H. and Trivedi, K. S.: Queueing Networks and Markov Chains, Wiley-Interscience, (1998).

3. Chirivella V. and Alcover R.: A New Reliability Model for Interconnection Networks, in Proceedings of EuroPar 2000, pp. 909-917.

4. Dally, W. J., and Aoki, H., Deadlock-Free Adaptive Routing in Multicomputer Networks Using Virtual Channels, IEEE TPD Systems, vol.4, no.4, pp.466-477, April 1993.

5. Duato, J.: A Theory of Fault Tolerant Routing in Wormhole Networks, IEEE TPD Systems (1997), vol.8, no.8, 790-802.

6. Dugan, J.B. and Trivedi, K. S.: Coverage Modeling for Dependability Analysis of Fault-Tolerant Systems, IEEE Transactions on Computers (1989), vol.38, no.6, 775-787.

7. Gaughan P. T. and Yalamanchili S.: A Family of Fault-Tolerant Routing Protocols for Direct Multiprocessor Networks, IEEE TPD Systems (1995), vol.6, no.5, 482-497. 
8. Scott, S. L. and Thorson, G.: The Cray 3TE Networks: Adaptive Routing on High Performance 3D Torus, in Proc. of Hots Interconnects IV, August 1996.

9. Vaidya, A. S., Das, R. C. and Sivasubramaniam A.: A Testbed for the Evaluation of Fault- Tolerant Routing in Multiprocessor Interconnection Networks, IEEE TPD Systems (1999), vol.10, no.10, 1052-1081. 